\title{
Preface for special section from FLOPS 2014
}

\author{
MICHAEL CODISH \\ Ben-Gurion University of the Negev \\ Department of Computer Science, Beer Sheva, Israel \\ (e-mail: mcodish@cs.bgu.ac.il) \\ EIJIRO SUMII \\ Tohoku University \\ Graduate School of Information Sciences, Sendai, Japan \\ (e-mail: sumii@ecei.tohoku.ac.jp)
}

The 12th International Symposium on Functional and Logic Programming was held in Kanazawa, Japan, June 4-6, 2014. The aim of the Functional and Logic Programming series of conferences is to bring together researchers interested in declarative programming including functional programming and logic programming. The series aims to promote cross-fertilization and integration between the two paradigms and it is traditionally organized and held in Japan.

For Functional and Logic Programming 2014, the call for papers mentioned that two to four of the best papers in each of the two areas would be invited for inclusion in a designated Functional and Logic Programming section within the Journal of Functional Programming and the journal Theory and Practice of Logic Programming.

After the conference the program committee selected five outstanding papers for journal publication: two for publication in Theory and Practice of Logic Programming, and three for publication in Journal of Functional Programming. The first two papers appeared in Theory and Practice of Logic Programming 16:2 (March 2016). The other three papers appear in this volume.

In the first paper, "Generating Constrained Random Data with Uniform Distribution", Claessen, Duregård and Pałka present a technique to automatically derive test data generators from a given executable predicate representing the set of values of interest for the generation. The describe how they make this process more efficient and present experimental evaluation.

In the second paper, "Type-Based Amortized Resource Analysis with Integers and Arrays", Hoffmann and Shao present a new approach to resource-bound analysis for numeric and heap-manipulating programs based on type-based amortized resource analysis. The technique is demonstrated for a first-order ML-like language with unsigned integers and arrays.

In the third paper, "A Characterization of Lambda-terms Transforming Numerals", Parys shows that $\lambda$-terms of each fixed type can be described by a finite piece of information (a set of intersection types) and by a vector of natural numbers. 
Parys continues to show that this description is compositional and demonstrates interesting consequences of this fact.

We would like to thank the authors and the reviewers for their outstanding efforts. We also gratefully acknowledge the assistance of the two journal editors: John Gallagher, the editor in charge of Rapid publications in the journal of Theory and Practice of Logic Programming, and Jeremy Gibbons, the co-Editor-in-Chief of the Journal of Functional Programming. 